\title{
BMJ Open Effect of early palliative care for patients with glioblastoma (EPCOG): a randomised phase III clinical trial protocol
}

\author{
Heidrun Golla (D) , ${ }^{1}$ Charlotte Nettekoven, ${ }^{1,2}$ Claudia Bausewein, ${ }^{3}$ \\ Jörg-Christian Tonn, ${ }^{4}$ Niklas Thon, ${ }^{4}$ Berend Feddersen, ${ }^{3}$ Oliver Schnell, ${ }^{5}$ \\ Christopher Böhlke, ${ }^{6}$ Gerhild Becker, ${ }^{6}$ Roman Rolke, ${ }^{7,8}$ Hans Clusmann, ${ }^{8,9}$ \\ Ulrich Herrlinger, ${ }^{10}$ Lukas Radbruch, ${ }^{11}$ Hartmut Vatter, ${ }^{8,12}$ Erdem Güresir, ${ }^{8,12}$ \\ Stephanie Stock, ${ }^{13}$ Dirk Müller, ${ }^{13}$ Daniele Civello, ${ }^{13}$ Irini Papachristou, ${ }^{14}$ \\ Martin Hellmich, ${ }^{15}$ Stefanie Hamacher, ${ }^{15}$ Raymond Voltz, ${ }^{1,8,14,15}$ \\ Roland Goldbrunner, ${ }^{2,8,16}$ EPCOG study group
}

To cite: Golla H, Nettekoven C, Bausewein C, et al. Effect of early palliative care for patients with glioblastoma (EPCOG): a randomised phase III clinical trial protocol. BMJ Open 2020;10:e034378. doi:10.1136/ bmjopen-2019-034378

- Prepublication history and additional material for this paper are available online. To view these files, please visit the journal online (http://dx.doi org/10.1136/bmjopen-2019034378).

Received 19 September 2019 Revised 15 November 2019 Accepted 15 November 2019

Check for updates

(C) Author(s) (or their employer(s)) 2020. Re-use permitted under CC BY-NC. No commercial re-use. See rights and permissions. Published by BMJ.

For numbered affiliations see end of article.

Correspondence to Dr Heidrun Golla;

heidrun.golla@uk-koeln.de

\section{ABSTRACT}

Introduction Randomised controlled trials (RCTs) have shown a positive effect of early integration of palliative care (EIPC) in various advanced cancer entities regarding patients' quality of life $(\mathrm{Q} o \mathrm{~L})$, survival, mood, caregiver burden and reduction of aggressiveness of treatment near the end of life. However, RCTs investigating the positive effect of EIPC for patients suffering from glioblastoma multiforme (GBM) are lacking. After modelling work identifying the specific needs of GBM patients and their caregivers, the aim of this study is to investigate the impact of EIPC in this particular patient group.

Methods and analysis The recruitment period of this multicenter RCT started in May 2019. GBM patients $(n=214)$ and their caregivers will be randomly assigned to either the intervention group (receiving proactive EIPC on a monthly basis) or the control group (receiving treatment according to international standards and additional, regular assessment of $\mathrm{QD}$ ('optimised' standard care)).

The primary outcome is QoL assessed by subscales of the Functional Assessment of Cancer Therapy for brain tumour (FACT-Br) from baseline to 6 months of treatment. Secondary outcomes are changes in QoL after 12 (end of intervention), 18 and 24 months (end of follow-up), the full FACT-Br scale, patients' palliative care needs, depression/anxiety, cognitive impairment, caregiver burden, healthcare use, costeffectiveness and overall survival.

Ethics and dissemination The study will be conducted in accordance with the Declaration of Helsinki and has been approved by the local ethics committees of the University Clinics of Cologne, Aachen, Bonn, Freiburg and Munich (LMU). Results of the trial will be submitted for publication in a peer-reviewed, open access journal and disseminated through presentations at conferences.

Trial registration number German Register for Clinical Studies (DRKS) (DRKS00016066); Pre-results.

\section{Strengths and limitations of this study}

For the first time the effect of a well-articulated, manualised palliative care intervention is being studied in a randomised controlled trial in a patient group (glioblastoma multiforme) with a wide range of neurological, psychological and psychiatric symptoms.

- Patients and caregivers are studied thoroughly over a period of 24 months, allowing for an observation period throughout the entire disease phase (for the majority of the patients).

- Missing values will be minimised by joint and proxy assessments should the patients' self-assessment not be possible.

- Permission of proxy assessment may cause a certain bias.

- Blinding of the researcher conducting the outcome measurements depends mainly on discretion of study participants.

\section{INTRODUCTION}

\section{Background}

Glioblastoma multiforme (GBM) is a highly malignant primary brain tumour. It can be regarded as a model for a rapidly progressive cancer with a wide range of fast-developing, life-changing symptoms encompassing neurological, psychological, psychiatric symptoms, as well as unpredictable personality changes, leading to loss of autonomy. ${ }^{1-8}$ The burden is mainly related to the psychosocial dimension in contrast to other advanced cancer entities. ${ }^{9-11}$

Previous randomised controlled trials (RCTs) in various advanced cancer groups other than GBM have shown a positive effect 
of early integration of palliative care (EIPC) in these patient groups. ${ }^{12-18}$ The palliative care (PC) approach aims to relieve physical, psychological, social and spiritual symptoms and problems as well as to enhance the quality of life (QoL) of the patients and of their next to kin (unit of care) suffering from progressive incurable diseases. According to the WHO definition, PC uses a team approach and should be applied early in the course of illness. ${ }^{19}$ Overall, the positive effects of EIPC in the aforementioned studies ${ }^{12-18}$ were improvement of QoL, survival, mood, caregiver burden and reduction of aggressiveness of treatment near end of life (EOL). The 'EVI project' in Germany evaluates whether EIPC can be implemented into the everyday clinical practice of Comprehensive Cancer Centers. ${ }^{20}$ Patients included in that study are those suffering from non-small cell lung cancer, metastatic oesophageal carcinoma, metastatic stomach carcinoma and non-endocrine pancreas carcinoma but without a special focus on GBM. A cohort study on GBM patients ${ }^{21} 22$ found a positive impact of a multiprofessional and interdisciplinary home care team on GBM patients and on the hospitalisation rate. Also, the costs of the hospitalisation were significantly reduced compared with standard care. However, PC professionals were not involved, just as in RCTs testing rehabilitative and supportive care interventions in patients with highgrade gliomas and their caregivers. ${ }^{23}$ In summary, we conclude that EIPC has the potential to benefit patients with several oncological entities as well as their caregivers. Nevertheless, the effect of EIPC on GBM patients in their highly complex situation and on their caregivers has yet to be evaluated. We, therefore, aim to investigate whether the benefit of EIPC on systemic cancers ${ }^{12-18}$ can also be found and confirmed in GBM.

\section{Rationale}

Temel $e t a l^{12}$ found in their landmark RCT involving patients with non-small cell lung cancer that EIPC leads to improvement of QoL and depression, to a reduction in aggressive treatment near the EOL and even to an increased life expectancy. Similar results could be confirmed for other advanced cancers. ${ }^{13-18}$ However, there are no data about the effect of EIPC in patients with GBM and they are less likely to receive PC than other cancer patients. ${ }^{124}$ Their disease-specific needs are probably not met by a 'one-size-fits-all' generic PC approach as they suffer from unique, serious, fast-developing, neuropsychiatric life-changing symptoms with a high caregiver burden ${ }^{1-11}$ as also shown in own studies. ${ }^{1-3} 7$ Therefore, this study aims to evaluate whether EIPC is efficacious (1) in a patient group with very different needs, (2) in medical disciplines not very experienced in PC yet, such as neurosurgery/neuro-oncology and (3) within the practicability of the German healthcare system. Following Temel et al, ${ }^{12}$ we will use QoL as a primary endpoint and an EIPC team as the intervention adapted to the special needs of GBM patients (PC physician and PC social worker instead of PC physician and PC nurse). For the individual patient we expect that EIPC results in better symptom control and improved QoL, reduced caregiver burden, improved co-ordination of care, more efficient and appropriate use of healthcare services and less unnecessary emergency admissions at the EOL. EIPC will help to clarify treatment plans early in the course of the disease which are often non-existent in this patient group. ${ }^{6}$ EIPC is constructed in a way that (1) the visits with specialised PC are face to face and/or via telephone, (2) specialised PC should serve as a mediator between patients and already existing services and (3) the caregivers' view and assessment plays a crucial role especially if patients are unable to self-assess any longer. All these strategies are resource-oriented, focus on clinical reality and practicability and have an economic impact. If this design proves to be successful, it could be used as a future strategy improving the level of care for GBM patients throughout the course of the disease until the EOL in Germany.

In spite of the positive results of EIPC in systemic cancer $^{12-18}$ we do not know how EIPC affects GBM patients, if such an effect will be sustainable and when to integrate PC in the care of this patient group. Therefore, our proposed design involves a wide range of GBM patients (initial diagnosis, recurrence, rural and urban areas) for a long period of time (12 months intervention, 12 months follow-up), in most cases, probably until death (median survival of GBM patients is between 15 and 17 months) ${ }^{25-27}$ This means that EIPC effects on this patient group will be studied comprehensively and will answer our study questions using a prospective clinical study design. What justifies a confirmatory clinical trial at this time point? Following the Medical Research Council (UK)in its current version from $2008,{ }^{28}$ we believe that the development phase of our complex intervention can be based on numerous studies including own modelling work identifying the specific needs of GBM patients and their caregivers ${ }^{1-11}$ and also the efficacy of EIPC. ${ }^{12-18} \mathrm{~A}$ potential intervention for GBM patients can be modelled on this existing evidence and our clinical experience. We have conducted two feasibility studies on prospective ongoing patient-reported outcome measurement (PROM) data collection from diagnosis to death ${ }^{2}$ and on assessing PC needs of GBM patients and caregiver burden. ${ }^{3}$ Our results demonstrated that prospective ongoing PROM data collection is feasible but that the importance of caregivers' external assessment increases ${ }^{23}$ with disease progression and that this did not bias our results significantly. ${ }^{2}$ Therefore, in this trial, we will involve proxies in the assessment, if patients should be unable to participate themselves. Our previous studies also demonstrated the challenges of recruitment and attrition in this patient group with a recruitment rate of almost $30 \%$ of all GBM patients in the field and a considerably high attrition rate of $10 \%-79 \%^{23}$ depending on the presence of assessment personnel. High attrition in PC trials is wellknown, ${ }^{29-31}$ thus to assure sufficient recruitment rates, a study nurse will at least partly be engaged at the neurosurgery/neuro-oncological departments at each site 
(usually neurosurgery, depending on the study site neurooncology (Bonn)). The study nurse will carefully identify all eligible patients (inpatient/outpatient), provide and explain trial information to all potential study participants (patients and respective caregivers), contact the responsible neurosurgery/neuro-oncology physician, organise the contacts between eligible patients and the neurosurgery/neuro-oncology physician and guide study participants through the clinical trial process. Our own experiences and results ${ }^{1-37}$ combined with the results of other existing studies ${ }^{4-69102122}$ lead to the next step of evaluating EIPC intervention for GBM patients including an assessment of efficacy. ${ }^{12-1723}$

The study period is from November 2018 to October 2023, while patient recruitment began in May 2019 and the last patient will be visited in April 2023.

\section{Objective}

\section{Primary objective}

The primary objective of this trial is to determine the efficacy of proactive early specialised PC tailored to patients with GBM to improve QoL. The changes in patients' QoL will be measured by the Trial Outcome Index (TOI, see the Measurements section) from baseline to 6 months.

It has been controversially discussed whether QoL is an appropriate measurement in PC and EOL care. ${ }^{32}$ However, QoL measures are widely used in PC studies, including the landmark study by Temel et al. ${ }^{12}$ The first statement about PC in the WHO definition is 'Palliative care is an approach that improves the QoL of patients and their families...', so changes in QoL are of crucial importance to evaluate a PC intervention. Temel et al ${ }^{12}$ measured QoL, defined as their primary endpoint, using the validated Functional Assessment of Cancer Therapy (FACT). As our study is strongly influenced by the study of Temel $e t a l,{ }^{12}$ we also chose QoL as primary endpoint but using the specific module for brain tumour patients (FACT-Br). ${ }^{33}$

\section{Secondary objectives}

Secondary endpoints will be changes in patients' QoL measured by the TOI from baseline to 12 months (end of intervention), at 18 and 24 months of follow-up (to evaluate maintenance/sustainability of effect), full FACT-Br scale, ${ }^{33}$ patients' PC needs, patients' depression and anxiety, patients' cognitive impairment, caregiver burden, each outcome measurement being validated and cost-effectiveness (costs per FACT-Br-unit) from the societal perspective including direct medical and direct nonmedical costs. ${ }^{34}$ Moreover, data on overall survival and compliance will be collected.

\section{METHODS}

\section{Trial design and study setting}

The study is a multicenter, randomised, confirmatory, phase III, rater-blinded, controlled, parallel-group, clinical trial testing the efficacy of proactive early specialised PC tailored to patients with GBM to improve QoL.

The trial is conducted at the Departments of Palliative Care and the Departments of Neurosurgery of the University Hospitals of Cologne, Aachen, Bonn (here, additionally Department of Neurooncology), Freiburg and Munich, Germany (list of study sites: please see DRKS00016066).

\section{Recruitment}

The neurosurgery/neuro-oncology study nurse of each site will see all patients potentially fulfilling predefined inclusion criteria (see the Eligibility criteria section) (outpatient and inpatient). The study nurse will provide information material about the study to each potentially eligible patient and caregiver. These patients and caregivers will be referred to the treating neurosurgery/ neuro-oncology physicians who will make the final decision on eligibility, explain the study and obtain informed written consent prior to enrolment or document why GBM patients or their caregivers could not be recruited into the study (inclusion criteria do not fit or refusal of study participation). Time between diagnosis of first or recurrent GBM and study inclusion may not exceed 4 weeks. The neurosurgery/neuro-oncology study nurse will co-ordinate further steps of the study (eg, baseline assessment, randomisation, start and further steps of intervention/ follow-up). After obtaining written informed consent (see online supplementary file 1 for model consent form), the baseline visit will be carried out by the 'assessment researcher' (researcher being responsible for the assessment) either on the ward, in the outpatient clinic or at patients' home/whereabouts (see figure 1). Afterwards, patients will be randomly assigned to either the control or the EIPC group (see the Randomisation section).

\section{Assessment visits and data collection}

At all study sites, the blinded assessment researcher will collect patients' and caregivers' data during personal meetings at the patient's home/whereabouts, commencing at study inclusion (baseline) and then every 3 months until month 12. These visits will be scheduled slightly delayed to routine clinical visits (time frame allowed +2 weeks), where patients of both groups will receive an 'optimised' standard care (OSTC) (for more information, see the Treatment arms section). Face-to-face data collection at patient's home/whereabouts will be pursued to minimise the degree of burden for study participants (they do not have to come in for another clinic appointment), to establish a confidential relationship and to increase the number of patients' self-assessment as strategies can be used which are not possible during a telephone assessment. To promote participant retention and to complete follow-up, caregivers may complete the questionnaires as an external assessment should patients feel overburdened.

Patients at all study sites live not only in the respective town but also in larger catchment areas. In our study, we will intentionally include patients from these catchment 


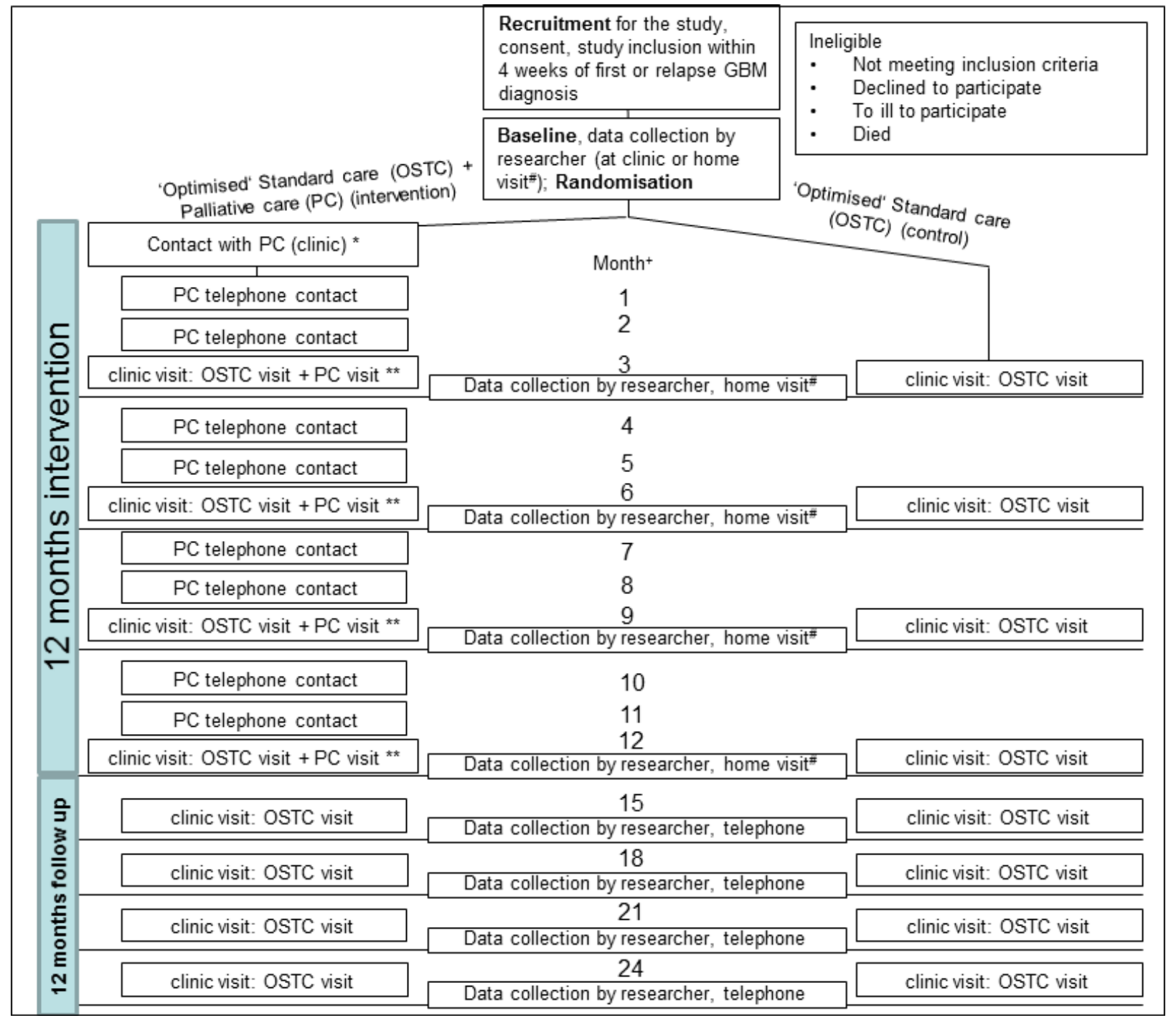

Figure 1 Trial flow chart. "Home visit means: the assessment researcher visits the patient for data collection at home or his/ her whereabouts. ${ }^{+}$All visits are allowed to be scheduled within a time frame of \pm 1 week except for the assessment visits for data collection at patient's home/whereabouts every 3 months. These assessment visits must be scheduled after the respective clinic visit (and PC visit, intervention group, only) within a time frame of +2 weeks. *After randomisation and before first PC contact by telephone (no later than 4 weeks after study inclusion): first contact of PC physician and PC social worker (EIPC team) with patients/caregivers to introduce themselves (not yet an EIPC visit but solely serves the purpose of getting acquainted with each other before the first EIPC contact by telephone). ${ }^{*}$ If patient is too ill for clinic visit telephone contact with EIPC team instead. EIPC, earlyintegration of palliative care; GBM, glioblastoma multiforme; OSTC, optimised standard care; PC, palliative care.

areas as we want to offer all patients treated at the study sites the opportunity to participate. Therewith, the study covers the true distribution of GBM patients in rural and urban areas and the results will reflect 'real-world' settings of GBM patients in Germany. This inevitably entails time-consuming and cost-consuming data collection by the assessment researcher (travel times, timeconsuming assessments due to severe disease). After the end of the intervention, patients and/or caregivers will be contacted by the already familiar assessment researcher via telephone 3-monthly up to 12 months to collect data on patients' and caregivers' current status or place and date of death (see figure 1). We opted for telephone calls at this point in time to keep study costs to a minimum.
Data collected beyond 6 months of the intervention and after the end of the intervention will be used to evaluate the maintenance/sustainability of the effect. During the EIPC intervention, in the intervention group faceto-face PC visits (months $3,6,9,12$ ) and PC telephone contacts (months $1,2,4,5,7,8,10,11$ ) will be conducted according to a PC manual.

\section{Eligibility criteria}

Patients are eligible to participate in the EPCOG Trial if they have a newly diagnosed GBM within 4 weeks of diagnosis (histologically confirmed by biopsy or resection) as well as patients with a recurrent GBM within 4 weeks after diagnosis of recurrence (confirmed according to RANO 
Table 1 Key inclusion and exclusion criteria

\begin{tabular}{|c|c|c|}
\hline & Patients & Caregivers \\
\hline Inclusion criteria & $\begin{array}{l}\text { Patients with newly diagnosed GBM } \\
\text { (histologically confirmed by biopsy or resection) } \\
\text { within } 4 \text { weeks of diagnosis or } \\
\text { Patients with recurrent GBM within } 4 \text { weeks after } \\
\text { diagnosis of recurrence. }\end{array}$ & $\begin{array}{l}\text { Caregiving persons (relatives or other closely } \\
\text { related persons) of special importance for the } \\
\text { patients, that is, they live with them or have face- } \\
\text { to-face contact with them at least twice a week. } \\
\text { Note: Patients can also be included if no such } \\
\text { caregiver exists. }\end{array}$ \\
\hline Exclusion criteria & $\begin{array}{r}\square \text { Unwillingness to } \\
\text { Being lega } \\
\text { makes the patient or caregive } \\
\square \text { Any kind of dependency on the investiga } \\
\text { Held in an institutio }\end{array}$ & $\begin{array}{l}\text { abide by the protocol. } \\
\text { lly incapacitated. } \\
\text { hiatric condition that, in the opinion of the investigator, } \\
\text { er unsuitable for study participation. } \\
\text { tor or employed by the sponsor or investigator. } \\
\text { n by legal or official order. }\end{array}$ \\
\hline
\end{tabular}

${ }^{*}$ ECOG performance status ${ }^{51}$ : grade 0: fully active, able to carry on all pre-disease performance without restriction; grade 1: restricted in physically strenuous activity but ambulatory and able to carry out work of a light or sedentary nature, for example, light house work, office work; grade 2: ambulatory and capable of all self-care but unable to carry out any work activities, up and about more than $50 \%$ of waking hours; grade 3: capable of only limited self-care, confined to bed or chair more than $50 \%$ of waking hours; grade 4: completely disabled, cannot carry on any self-care, totally confined to bed or chair; grade 5: dead.

ECOG, Eastern Cooperative Oncology Group; GBM, glioblastoma mulitforme.

criteria or if clinical and/or radiological deterioration leads to a change in oncological treatment (eg, re-surgery/stereotactic biopsy, re-irradiation and/or change in chemotherapy protocol or dosage, which is not based on side effects like thrombocytopenia) as indicated by the local investigator). Moreover, a caregiving person (caregiver; relatives or other closely related persons) can be included if of special importance for the patients, that is, they live with them or have face-to-face contact with them at least twice a week. Of note, patients can also be included if no such caregiver exists or is willing to participate in the study. Further inclusion and exclusion criteria for patients and caregivers are shown in table 1 .

\section{Randomisation}

Patients who meet the eligibility criteria will be randomly assigned into the intervention or the control group. Randomisation will be done using a 24/7 readily accessible internet-based tool (ALEA; FormsVision BV, Abcoude, the Netherlands). Patients will be assigned to treatment groups (ratio 1:1) according to permuted blocks of varying length. Randomisation will be stratified by study site, time point of PC intervention (initial diagnosis or recurrence) and availability of a caregiver (ie, 20 strata altogether). Patients will be informed about their allocation to the trial arm by the study nurse.

\section{Blinding}

The randomisation will be carried out by an unblinded team member, for example, by the neurosurgery/neurooncology study nurse. Study participants will be asked not to tell the assessment researcher whether they are in the intervention or in the control group. The status of the researchers' blindness will be queried after each visit. The statistician performing the statistical analysis will be blinded as well.

\section{Treatment arms}

\section{'Optimised' standard care}

In the control group, GBM patients will receive OSTC that includes regular visits to the neurosurgery/neurooncology outpatient clinic every 3 months $( \pm 1$ week) as well as treatment and routine assessments following international standards. ${ }^{35}$ In addition, the 'optimisation' includes regular assessments of the patients' QoL measured using the FACT-Br. ${ }^{33}$ This allows the primary treating physicians to detect and react on patients' current needs in a timelier and more frequent manner, including, if necessary, the integration of existing PC structures which is also allowed explicitly in the control group (reactive approach) (see figure 2). This format is chosen to weaken the argument that additional care and attention alone is sufficient to improve QoL and reduce PC needs. ${ }^{36}$

\section{Proactive, early PC}

In the intervention group, in addition to the OSTC, patients will be in regular, structured contact (according to a PC manual) with specialised PC, irrespective of their current needs ('proactive') (for further details, see the Interventions section). 


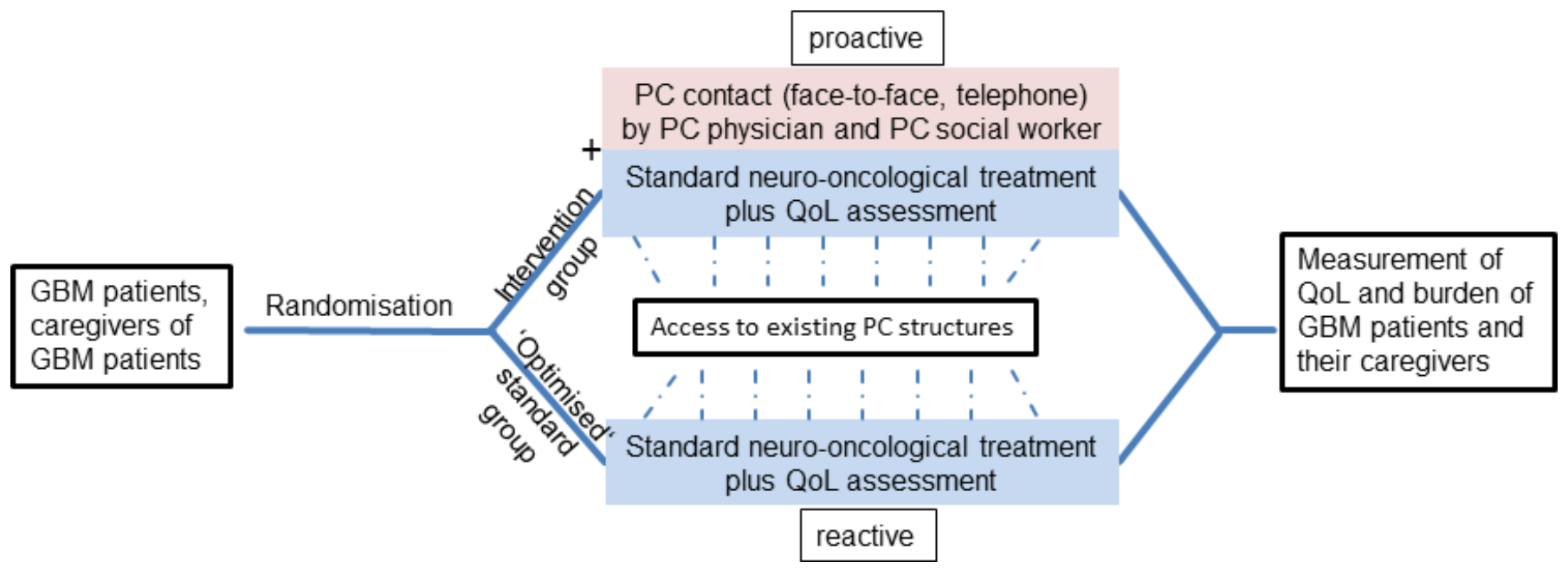

Figure 2 Intervention scheme. GBM, glioblastoma multiforme; PC, palliative care; QOL, quality of life.

\section{Interventions}

The EIPC intervention team consists of a PC physician and a PC social worker. The study plan schedules fixed face-to-face appointments with the EIPC team every 3 months, on the same day as the patients' appointment for their routine neurosurgery/neuro-oncology treatment at the clinic (feasible in clinical practice), that is, participants will not have to come to the clinic just because of the clinical trial. Between these quarterly visits, patients will be contacted monthly by the EIPC team by telephone. If patients are too ill for a clinic visit, a PC clinic contact may be compensated by a telephone PC contact or caregivers may be contacted instead. ${ }^{2}$

All staff members performing interventions will strictly follow a PC checklist/manual. Accordingly, the EIPC team will focus on pain and symptom management, psychosocial and spiritual support, assistance in treatment decisions and help in care planning during their fixed face-to-face/telephone contacts with study participants (see online supplementary file 2 ).

\section{Sample size calculation}

The sample size calculation was performed by the Institute of Medical Statistics and Computational Biology, University of Cologne for the primary outcome TOI. Here, we assume an effect size of 0.5 for the comparison of the experimental treatment versus control as previously found by Temel et al. ${ }^{12}$ Assuming a similar SD of 20 points for the change in TOI $\left(\approx 11.6\right.$ \{SD early palliative care group\}* ${ }^{*} 148$ \{range TOI\}/84\{range TOI Temel et $\left.a l^{12}\right\}$ ), this medium effect size corresponds to a difference of 10 points in TOI, that is, about 4 points $[\approx 10 * 56$ \{range sum of subscales\} $/ 148$ \{range TOI\}] for the sum of the FACT-G/Br subscales physical and function. According to Cella $e t a l,{ }^{37}$ a clinically relevant change for the sum of the FACT-G/Br subscales physical and function is derived as half the mean of the difference between ECOG PSR 0 to 1 and 1 to 2 , that is, $4 \approx([\{4.1+4.6\}$ $+\{3.1+2.7\}\} / 2) / 2$ points. Thus, by approximation, the expected effect size 0.5 corresponds to a clinically relevant change. The two-sample t-test requires 64 patients per treatment group to yield $80 \%$ power at two-sided significance level 5\% (Stata V.14.1, StataCorp; power two means). Thus, cautiously, 128 evaluable patients need to complete the trial. Accounting for up to $40 \%$ dropout, 214 patients need to be included and randomised. Nota bene, power may be further increased by taking a baseline-adjusted mixed model for repeated measures (MMRM) approach for statistical analysis. Specifically, assuming (1) one baseline measurement, (2) two follow-up measurements after randomisation (at 3 and 6 months) and (3) a correlation of 0.5 between repeated measurements, 128 evaluable patients may be sufficient to detect an effect size as small as 0.35 with $80 \%$ power. $^{38}$

\section{Measurements}

The primary outcome measure is the QoL as assessed by the FACT-Br following Temel $e$ al. ${ }^{12}$ The QoL will be analysed by the TOI ( 37 items scored $0-4$, range $0-148$ ) which is the sum of scores on the $\mathrm{Br}$ (Br1-21, NTX6, An10), and the physical well-being (GP1-7) and functional well-being (GF1-7) subscales of the FACT-Br-scale ${ }^{33}$ from baseline to 6 months of treatment.

The maintenance/sustainability of EIPC will be measured by the TOI from baseline to 12 (end of intervention), 18 and 24 months (follow-up).

Moreover, the following outcome measures will be assessed at baseline and every 3 months with secondary endpoints at months 6 and 12 (end of intervention), as well as at months 18 and 24 (follow-up after treatment):

- PC needs: Integrated Palliative Care Outcome Scale (IPOS)..$^{39} 40$

- Depression and anxiety: Hospital Anxiety and Depression scale (HADS). ${ }^{41} 42$

- Cognitive screening: Montreal Cognitive Assessment (MoCA). ${ }^{43-45}$

- Caregiver burden: Zarit Burden Interview, short version (12 questions; ZBI-12) ${ }^{46-48}$ adapted version according to Kühnel et al. $^{49}$

- Overall survival. 
- Use of healthcare and cost-effectiveness: type and number of contacts to healthcare structures; type, lengths and frequency of tumour therapies usual for GBM patients ${ }^{34}$ (see online supplementary file 3).

- Case and data reporting form (see online supplementary files 4-7).

\section{Data analysis plan}

The change in QoL (as assessed by the TOI) from baseline to 6 months after randomisation will be evaluated by a MMRM over time (ARH1-structured covariance matrix). To account for and assess the impact of attrition multiple imputation approaches are taken, accounting for proxy measures and assuming specific missingnessnot-at-random patterns. Time-to-event (eg, dropout or survival) distributions are summarised by the KaplanMeier method and compared by the (stratified) log-rank test. Secondary outcomes (ie, further time points and measures) are analysed along the same lines.

Subgroup analyses are done by study site, time point of PC intervention, availability of a caregiver and sex; interaction with treatment is investigated. No interim analysis is planned. The trial may be terminated prematurely if there are any relevant medical or ethical concerns, or if completing the trial is no longer practicable.

\section{Data monitoring}

A data monitoring committee is not applicable (non$\mathrm{AMG} /$ non-MPG clinical trial). An internal trial steering committee was formed to monitor the progress of the trial, manage and supervise all trial procedures on a regular basis and reach majority decisions on upcoming questions. The internal trial steering committee is advised by an independent, interdisciplinary and multidisciplinary scientific advisory board, whose members are involved in planning, guiding and evaluation of the trial.

\section{Patient and public involvement}

To assure patients' perspectives in the study question, design and implementation, we directly invited the patient support organisation 'German Brain Cancer Aid e.V.' for support with this study. They have declared their willingness to support us by commenting on the study proposal and helping with dissemination on patient platforms. Moreover, we involved patient representatives of the University Hospital of Cologne to comment on the study proposal.

\section{ETHICS AND DISSEMINATION \\ Ethical considerations}

Protocol amendments will be submitted to the ethics committee and signed by all authors of the trial protocol. Amendments will be published in the online registration of the trial and in the trial paper.

The Clinical Trials Centre Cologne (CTCC) is an external party that will monitor the study in a risk-adapted way and ensure that the study follows GCP, that is, that all participants give informed written consent and that studyrelated materials are handled correctly.

\section{Safety considerations}

We do not expect Adverse Events in this non-AMG/nonMPG clinical trial. GBM patients' death and worsening in general conditions is expected during the trial as intervention and follow-up extend over a period of time in which GBM patients are expected to die due to the given disease course. The EIPC intervention applied in this clinical trial has been proven for other disease entities-though in modified form-not to harm but rather to improve QoL, reduce depression and decrease aggressive treatment to the EOL. ${ }^{12-18}$ The EIPC intervention according to the PC checklist/manual is designed such that primary treating physicians will get a feedback on patients' / caregivers' complaints and the measures will be taken, so that they have the possibility to react to particularities unless not done by the EIPC team itself. In addition to this, the control group will also be 'optimised' by regularly measuring QoL using the FACT-Br. ${ }^{33}$ Primary treating physicians can directly act on distress becoming obvious during FACT-Br assessment (eg, items ticked with the worst score) and react on patients' specific needs also apart from planning tumour-specific treatment. In both groups, all available healthcare structures are allowed to be integrated into the patients' care, if needed, including existing PC structures. Apart from the regular use of the FACT-Br, ${ }^{33}$ study participants will be assessed every 3 months. Using the regular outcome measurements (FACT-Br, ${ }^{33}$ IPOS, ${ }^{39}{ }^{40}$ HADS, ${ }^{41}{ }^{42}$ ZBI-12) ${ }^{48}$ a distress score will be calculated every 6 months. Thereby potential distress will be detected. Forty-nine items (including the domains physical (pain, dyspnoea, vomiting and so on), psychological (anxiety, depression and so on), relationship (family, friends)) are regarded relevant for distress phenomena. Items ticked with the worst score possible are classified as critical, recoded items are summed up and the sum is divided by the number of answered items. Relevant items will be aggregated by category (by the principal coordinating investigator) and listed. The internal steering committee will regularly monitor any (aggregated) distress phenomena which can be extracted from the database.

\section{Dissemination plan}

Findings of this study will be disseminated broadly to participants, healthcare professionals, the public and other relevant groups. The study protocol and results of this study will be published open access in a peer-reviewed scientific journal and presented at national and international conferences.

\section{Confidentiality}

Patients and caregivers will be given a trial number so that personally identifying information cannot be linked to assessment or trial information. All investigational materials and data will be pseudonymised in accordance with data protection legislation before scientific processing. 


\section{Documentation and quality assurance}

All data relevant to the trial will be directly documented in the electronic case reporting form (eCRF) or soon after measurement by the investigator responsible (documentation of EIPC according to PC manual by PC physician and social worker). Entering data may be delegated to members of the trial team except for the assessment researcher who has to stay blinded.

The assessment researcher who visits the patients for data collection at home or their whereabouts will directly document the results of the outcome measurements in the database (eCRF). This comprises the data and case reporting forms (documenting the healthcare use), sociodemographic data, the caregiver form (ZBI12) and all patient forms (Fact-Br, IPOS, HADS) besides from the MoCA. The latter must be filled out on paper, so that only the results of each cognitive domain are entered into the eCRF. In the follow-up phase, the MoCA will be administered by telephone (T-MoCA) ${ }^{50}$, as no face-to-face assessment is planned during the follow-up phase. In case of technical failure (eg, missing internet connection), the assessment researcher will complete all measures on paper but transfer all acquired data into the eCRF right after the study appointment. The FACT-Br filled out during regular neurosurgery/neurooncology clinic visits (OSTC) will be kept in the patient's file. The database used, TrialMaster, is validated (OmniComm.com). Every correction made to the data is traceable. Only authorised persons have access to the programme and the data. Regular data backups will be made. The eCRFs are signed by the principal investigators of each trial centre.

Monitoring is conducted by the CTCC Cologne and will include written informed consent, as well as riskbased monitoring of all inclusion/exclusion criteria and source data. Other than initiation visits at the beginning of the study and close-out visits at the end of the study, monitoring visits will be scheduled based on the number of patients included at each study centre (first visit: after 15-20 patients or after 1 year).

\section{Data management}

The IT infrastructure and data management staff will be supplied by the CTCC. The trial database will be developed and validated before data entry according to standard operating procedures (SOPs) at the CTCC. The data management system is based on commercial trial software and stores the data in a database. All changes made to the data are documented in an audit trail. The trial software has a user and role concept that can be adjusted on a trialspecific basis. The database is integrated into a general IT-infrastructure and safety concept with a firewall and backup system. The data are backed up daily. At the study end or after premature termination after confirmed completion and cleaning of data, the database is locked and the data are exported for statistical analysis.

The data will be entered online at the trial sites. Plausibility checks are run during data entry, thereby detecting discrepancies immediately. The CTCC data management will conduct further checks (listed in a study-specific data review plan) for completeness and plausibility and will clarify any questions with the trial sites according to the SOPs via queries. These electronic queries have to be answered in a timely manner by the trial site.

\section{Author affiliations}

${ }^{1}$ Department of Palliative Medicine, Faculty of Medicine and University Hospital, University of Cologne, Cologne, Germany

${ }^{2}$ Department of General Neurosurgery, Faculty of Medicine and University Hospital, Center for Neurosurgery, University of Cologne, Cologne, Germany

${ }^{3}$ Department of Palliative Medicine, Faculty of Medicine and University Hospital, University of Munich (LMU), Munich, Germany

${ }^{4}$ Department of Neurosurgery, Faculty of Medicine and University Hospital, University of Munich (LMU), Munich, Germany

${ }^{5}$ Department of Neurosurgery, Faculty of Medicine and University Hospital, University of Freiburg, Freiburg, Germany

${ }^{6}$ Department of Palliative Medicine, Faculty of Medicine and University Hospital, University of Freiburg, Freiburg, Germany

${ }^{7}$ Department of Palliative Medicine, Faculty of Medicine and University Hospital, RWTH Aachen University, Aachen, Germany

${ }^{8}$ Faculty of Medicine and University Hospital, Center for Integrated Oncology Aachen Bonn Cologne Duesseldorf (CIO ABCD), University of Cologne, Cologne, Germany ${ }^{9}$ Department of Neurosurgery, Faculty of Medicine and University Hospital, RWTH Aachen University, Aachen, Germany

${ }^{10}$ Division of Clinical Neurooncology, Department of Neurology, Faculty of Medicine and University Hospital, University of Bonn, Bonn, Germany

${ }^{11}$ Department of Palliative Medicine, Faculty of Medicine and University Hospital,

University of Bonn, Bonn, Germany

${ }^{12}$ Department of Neurosurgery, Faculty of Medicine and University Hospital, University of Bonn, Bonn, Germany

${ }^{13}$ Faculty of Medicine and University Hospital, Institute for Health Economics and Clinical Epidemiology (IGKE), University of Cologne, Cologne, Germany

${ }^{14}$ Faculty of Medicine and University Hospital, Center for Clinical Studies (ZKS), University of Cologne, Cologne, Germany

${ }^{15}$ Faculty of Medicine and University Hospital, Institute of Medical Statistics and Computational Biology (IMSB), University of Cologne, Cologne, Germany

${ }^{16}$ Faculty of Medicine and University Hospital, Center for Health Services Research (ZVFK), University of Cologne, Cologne, Germany

Acknowledgements This trial is supported by Clinical Trials Centre Cologne (CTCC) Medical Faculty, University of Cologne by performing the Project Management, Data Base Development, Data Management and Monitoring. The EPCOG study group would like to thank the members of the Scientific Advisory Board: Dr rer. pol. Ute Karbach, deputy professorship, Faculty of Rehabilitation Sciences, Rehabilitation Sociology, University of Technology Dortmund, Germany; Professor Dr David Oliver, The Tizard Centre, University of Kent, Canterbury, UK; Professor Dr Martin JB Taphoorn, Department of Neurology, Leiden University Medical Center, Leiden, The Netherlands; Professor Dr Christian Rietz, Department of Educational Science, Faculty of Educational and Social Sciences, University of Education Heidelberg, Heidelberg, Germany.

Collaborators EPCOG study group. Aachen: Imke Bronger; Kerstin Dederichs; Hildegard Cavelius; Sonja Hiddemann; Norbert Krumm; Christina Thamm; Daniel Delev; Marita Kumschlies; Manuela Langheimer; Chuh-Hyoun Na. Bonn: Christiane Landwehr; Niklas Schäfer; Christina Schaub; Claudia Stratmann; Kirsten Hüning; Birgit Jaspers; Michaela Hesse. Cologne: Isabel Franke; Melanie Joshi; Simone Matte; Lena Dreher; Gina Fürtjes; Sophia Kochs; Moritz Lenschow; Patrick Melich; Catharina Schröter. Freiburg: Bianca-Ioana Blaß; Debora Cipriani; Dieter Henrik Heiland; Pamela Heiland; Nadja Jarc; Nicole Koch; Marcia Regina Machein; Nicolas Neidert; Mateo Farina Nunez; Jolanda Daniuk; Bianca Lauble; Tina Meer; Saskia Opitz. Munich: Birgit Haberland; Eva Lehmann; Karla Steinberger; Sabine Streitwieser; Christoph Barth; Stefanie Lietke; Christiane Zimmerer.

Contributors $\mathrm{HG}$ (principal coordinating investigator), CN, ClaB, J-CT, NT, BF, OS, ChrB, GB, RR, HC, UH, LR, HV, EG, SS, DM, DC, IP, MH, SH, RV and RG planned the study, wrote and corrected the study proposal, study proposal amendments, the 
study protocol and this manuscript. MH and SH provided statistical input to all study documents. $\mathrm{HG}, \mathrm{ChB}$ and $\mathrm{OS}$ form the internal steering committee.

Funding This work is supported by the Federal Ministry of Education and Research (BMBF), Germany, FKZ: $01 \mathrm{GY} 1703$.

Competing interests None declared.

Patient consent for publication Not required.

Ethics approval The trial will be conducted in compliance with this study protocol, the Declaration of Helsinki and Good Clinical Practice. The trial has been approved by the local ethics committees of the University Clinics of Cologne, Aachen, Bonn, Freiburg and Munich (LMU).

Provenance and peer review Not commissioned; externally peer reviewed.

Open access This is an open access article distributed in accordance with the Creative Commons Attribution Non Commercial (CC BY-NC 4.0) license, which permits others to distribute, remix, adapt, build upon this work non-commercially, and license their derivative works on different terms, provided the original work is properly cited, appropriate credit is given, any changes made indicated, and the use is non-commercial. See: http://creativecommons.org/licenses/by-nc/4.0/.

\section{ORCID iD}

Heidrun Golla http://orcid.org/0000-0002-4403-630X

\section{REFERENCES}

1 Ostgathe C, Gaertner J, Kotterba M, et al. Differential palliative care issues in patients with primary and secondary brain tumours. Support Care Cancer 2010;18:1157-63.

2 Golla H, Ale Ahmad M, Galushko M, et al. Glioblastoma multiforme from diagnosis to death: a prospective, hospital-based, cohort, pilot feasibility study of patient reported symptoms and needs. Support Care Cancer 2014;22:3341-52.

3 Seibl-Leven M, von Reeken C, Goldbrunner R, et al. Clinical routine assessment of palliative care symptoms and concerns and caregiver burden in glioblastoma patients: an explorative field study. $J$ Neurooncol 2018;138:321-33.

4 Sizoo EM, Pasman HRW, Dirven L, et al. The end-of-life phase of high-grade glioma patients: a systematic review. Support Care Cancer 2014;22:847-57.

5 Sterckx W, Coolbrandt A, Dierckx de Casterlé B, et al. The impact of a high-grade glioma on everyday life: a systematic review from the patient's and caregiver's perspective. Eur J Oncol Nurs 2013;17:107-17.

6 Sizoo EM, Pasman HRW, Buttolo J, et al. Decision-making in the end-of-life phase of high-grade glioma patients. Eur J Cancer 2012;48:226-32.

7 Bausewein C, Hau P, Borasio GDI, et al. How do patients with primary brain tumours die? Palliat Med 2003;17:558-9.

8 Singer S, Roick J, Danker H, et al. Psychiatric co-morbidity, distress, and use of psycho-social services in adult glioma patients - a prospective study. Acta Neurochir 2018;160:1187-94.

9 Renovanz M, Hechtner M, Janko M, et al. Factors associated with supportive care needs in glioma patients in the neuro-oncological outpatient setting. J Neurooncol 2017;133:653-62.

10 Renovanz M, Hickmann A-K, Coburger J, et al. Assessing psychological and supportive care needs in glioma patientsfeasibility study on the use of the Supportive Care Needs Survey Short Form (SCNS-SF34-G) and the Supportive Care Needs Survey Screening Tool (SCNS-ST9) in clinical practice. Eur J Cancer Care (Engl) 2018;27. doi:10.1111/ecc.12598. [Epub ahead of print: 24 Oct 2016].

11 Renovanz M, Maurer D, Lahr H, et al. Supportive care needs in glioma patients and their caregivers in clinical practice: results of a multicenter cross-sectional study. Front Neurol 2018;9:763.

12 Temel JS, Greer JA, Muzikansky A, et al. Early palliative care for patients with metastatic non-small-cell lung cancer. $N$ Engl J Med 2010;363:733-42.

13 Bakitas MA, Tosteson TD, Li Z, et al. Early versus delayed initiation of concurrent palliative oncology care: patient outcomes in the enable III randomized controlled trial. J Clin Oncol 2015;33:1438-45.

14 Zimmermann C, Swami N, Krzyzanowska M, et al. Early palliative care for patients with advanced cancer: a cluster-randomised controlled trial. The Lancet 2014;383:1721-30.

15 Higginson IJ, Bausewein C, Reilly CC, et al. An integrated palliative and respiratory care service for patients with advanced disease and refractory breathlessness: a randomised controlled trial. Lancet Respir Med 2014;2:979-87.
16 Dyar S, Lesperance M, Shannon R, et al. A nurse practitioner directed intervention improves the quality of life of patients with metastatic cancer: results of a randomized pilot study. J Palliat Med 2012;15:890-5.

17 Temel JS, Greer JA, El-Jawahri A, et al. Effects of early integrated palliative care in patients with lung and Gl cancer: a randomized clinical trial. J Clin Oncol 2017;35:834-41.

18 El-Jawahri A, Greer JA, Pirl WF, et al. Effects of early integrated palliative care on caregivers of patients with lung and gastrointestinal cancer: a randomized clinical trial. Oncologist 2017;22:1528-34.

19 World Health Organization. WHO definition of palliative care. Available: http://www.who.int/cancer/palliative/definition/en/ [Accessed 9 Sept 2019].

20 Meffert C, Gaertner J, Seibel K, et al. Early palliative care-health services research and implementation of sustainable changes: the study protocol of the EVI project. BMC Cancer 2015;15:443.

21 Pace A, Di Lorenzo C, Capon A, et al. Quality of care and rehospitalization rate in the last stage of disease in brain tumor patients assisted at home: a cost effectiveness study. J Palliat Med 2012;15:225-7.

22 Pompili A, Telera S, Villani V, et al. Home palliative care and end of life issues in glioblastoma multiforme: results and comments from a homogeneous cohort of patients. Neurosurg Focus 2014;37:E5.

23 Piil K, Juhler M, Jakobsen J, et al. Controlled rehabilitative and supportive care intervention trials in patients with high-grade gliomas and their caregivers: a systematic review. BMJ Support Palliat Care 2016:6:27-34.

24 Walbert T. Integration of palliative care into the neuro-oncology practice: patterns in the United States. Neurooncol Pract 2014;1:3-7.

25 Chinot OL, Wick W, Mason W, et al. Bevacizumab plus radiotherapytemozolomide for newly diagnosed glioblastoma. $N$ Engl J Med 2014;370:709-22.

26 Stupp R, Mason WP, van den Bent MJ, et al. Radiotherapy plus concomitant and adjuvant temozolomide for glioblastoma. $N$ Engl J Med 2005;352:987-96.

27 Stupp R, Taillibert S, Kanner AA, et al. Maintenance therapy with tumor-treating fields plus temozolomide vs temozolomide alone for glioblastoma: a randomized clinical trial. JAMA 2015;314:2535-43.

28 Craig P, Dieppe P, Macintyre S, et al. Developing and evaluating complex interventions: the new medical Research Council guidance. Int J Nurs Stud 2013;50:587-92.

29 Hui D, Glitza I, Chisholm G, et al. Attrition rates, reasons, and predictive factors in supportive care and palliative oncology clinical trials. Cancer 2013;119:1098-105.

30 Jordhøy MS, Kaasa S, Fayers $\mathrm{P}$, et al. Challenges in palliative care research; recruitment, attrition and compliance: experience from a randomized controlled trial. Palliat Med 1999;13:299-310.

31 Addington-Hall JM, MacDonald LD, Anderson HR, et al. Randomised controlled trial of effects of coordinating care for terminally ill cancer patients. BMJ 1992;305:1317-22.

32 Evans CJ, Harding R, Higginson IJ, et al. 'Best practice' in developing and evaluating palliative and end-of-life care services: a meta-synthesis of research methods for the MORECare project. Palliat Med 2013;27:885-98.

33 Thavarajah N, Bedard G, Zhang L, et al. Psychometric validation of the functional assessment of cancer therapy-brain (FACT-Br) for assessing quality of life in patients with brain metastases. Support Care Cancer 2014;22:1017-28.

34 Müller D, Stock S, Dintsios C-M, et al. [Checklist for the development and assessment of cost-of-illness studies]. Gesundheitswesen 2018;80:744-53.

35 Weller M, van den Bent M, Hopkins K, et al. EANO guideline for the diagnosis and treatment of anaplastic gliomas and glioblastoma. Lancet Oncol 2014;15:e395-403.

36 Jatoi A. How important is palliative care? Curr Oncol Rep 2011;13:252-4.

37 Cella DF, Tulsky DS, Gray G, et al. The functional assessment of cancer therapy scale: development and validation of the general measure. J Clin Oncol 1993;11:570-9.

38 Machin D. Sample size tables for clinical studies. 3rd edn. Hoboken, NJ: Wiley-Blackwell. Chichester, West, Sussex, UK:section 6.2, 2008.

39 Hearn J, Higginson IJ. Development and validation of a core outcome measure for palliative care: the palliative care outcome scale. palliative care core audit project Advisory group. Qual Health Care 1999;8:219-27.

40 Bausewein C, Fegg M, Radbruch L, et al. Validation and clinical application of the German version of the palliative care outcome scale. J Pain Symptom Manage 2005;30:51-62. 
41 Bjelland I, Dahl AA, Haug TT, et al. The validity of the hospital anxiety and depression scale. An updated literature review. J Psychosom Res 2002;52:69-77.

42 Zigmond AS, Snaith RP. The hospital anxiety and depression scale. Acta Psychiatr Scand 1983;67:361-70.

43 Olson R, Tyldesley S, Carolan $\mathrm{H}$, et al. Prospective comparison of the prognostic utility of the mini mental state examination and the Montreal cognitive assessment in patients with brain metastases. Support Care Cancer 2011;19:1849-55.

44 Olson RA, Iverson GL, Carolan $\mathrm{H}$, et al. Prospective comparison of two cognitive screening tests: diagnostic accuracy and correlation with community integration and quality of life. $J$ Neurooncol 2011;105:337-44.

45 Renovanz M, Reitzug L, Messing L, et al. Patient reported feasibility and acceptance of Montreal cognitive assessment (MoCA) screening pre- and postoperatively in brain tumour patients. $J$ Clin Neurosci 2018;53:79-84.

46 Zarit SH, Reever KE, Bach-Peterson J. Relatives of the impaired elderly: correlates of feelings of burden. Gerontologist 1980;20:649-55.
47 Bédard M, Molloy DW, Squire L, et al. The Zarit burden interview: a new short version and screening version. Gerontologist 2001;41:652-7.

48 Higginson IJ, Gao W, Jackson D, et al. Short-form Zarit caregiver burden interviews were valid in advanced conditions. J Clin Epidemiol 2010;63:535-42.

49 Kühnel M, Ramsenthaler C, Bausewein C, et al. Validierung der 7-Item Kurz-Version des Zarit Burden Interviews-Ein Fragebogen zur Erhebung der Belastung von Angehörigen von Palliativpatienten

50 Pendlebury ST, Welch SJV, Cuthbertson FC, et al. Telephone assessment of cognition after transient ischemic attack and stroke: modified telephone interview of cognitive status and telephone Montreal cognitive assessment versus face-to-face Montreal cognitive assessment and neuropsychological battery. Stroke 2013;44:227-9.

51 Oken MM, Creech RH, Tormey DC, et al. Toxicity and response criteria of the eastern cooperative Oncology Group. Am J Clin Oncol 1982;5:649-56. 